\title{
Optimising the time-cost-quality (TCQ) trade-off in offshore wind farm project management with a genetic algorithm (GA)
}

\author{
Gloria Yushan Liu, Eric Wai Ming Lee and Richard Kwok Kit Yuen \\ Department of Architecture and Civil Engineering, City University of Hong Kong, Hong Kong, People's Republic of China
}

\begin{abstract}
Time, cost and quality are major concerns in construction project management. To achieve a balance between time-cost and time-quality, a trade-off problem among time-cost-quality (TCQ) is proposed for optimisation by the application of a genetic algorithm (GA). A GA attempts to minimise a fitness function that describes the objective to be achieved. The fitness function is specifically designed according to the nature and characteristics of the construction project. By inputting the project parameters, the fitness function should be able to provide a balance between the time, cost and quality of the project. This study applied a GA to strategically search for the best project parameters for an offshore wind farm project to achieve a more accurate prediction for construction time, cost and quality of the project in the pre-construction stage. A series of practical mathematical models are developed through a review of previous studies based on specific merits, and a real offshore wind farm project is studied to identify and verify the applicability and viability of the mathematical models. After the process of optimisation, the results show that the output data is very close to the actual case in terms of construction time, cost and quality.
\end{abstract}

KEYWORDS Offshore wind farm project; time-cost-quality (TCQ) trade-off; construction management; genetic algorithm (GA) CONTACT Gloria Yushan Liu@yushanliu3-c@my.cityu.edu.hk

Received 23 May 2019

\section{Introduction}

The requirement for the generation of sustainable energy has increased rapidly in recent years as the severe and irreversible environmental costs of traditional methods of energy generation have been concerned. New green energy development is now coming to the forefront, with an unprecedented level of application. After the first conference about an offshore wind farm held in Copenhagen in 2005, the world began to pay more attention to wind power generation because it is renewable and free from pollution. Furthermore, the benefits are even more pronounced in an offshore wind farm generation system (Zhixin et al., 2009). Unlike traditional methods of power generation such as fossil fuels, green energy including hydro, biomass, wind and solar power is renewable and sustainable (Klugmann-Radziemska, 2014). Among these types of green energy, hydro and biomass power have potential production limitations but wind and solar power are abundant and infinite. Wind power is relatively easy to generate and transfer, and it is thus widely recognised as a reliable and viable method for electricity production. Wind farms, as a new type of power generation, are widely applied worldwide and have developed rapidly in China since 2006 with both on-land and offshore applications. Compared to on-land wind farms, offshore wind farms occupy less land which allows fewer environmental limitations while the wind resources are more sufficient (Zhixin et al., 2009). However, offshore wind farms have some serious negative impacts on marine environment, such as on marine mammals, plants, and even on the landscapes and geological conditions (Carstensen et al., 2006). A conflict develops between the increasing need for electricity in human's lives and the demand to reduce these negative effects on the marine environment. More complicated and advanced techniques are applied to ensure a sufficient amount of electricity which can be generated with fewer wind farms, and results in the use of larger capacity turbines and higher voltage cables, to increase the generation efficiency of wind power and to reduce the electricity loss during transport. A more advanced technique requires a more precise installation and this leads to a more difficult and expensive construction process. An offshore wind farm project, as a large-scale one-time project, contains many uncertainties and no correct measures or amendments are allowed after construction. Therefore, the planning and budgeting processes are essential to avoid unforeseen problems and predictable errors that may happen during construction. One of the core objectives of construction planning, which consists of the pre-contract construction management, is to reach a perfect balance among construction time, cost and quality. This study proposes a systematic formulation to simulate the best circumstances in terms of project time, cost and quality simultaneously to ensure that the planning stage is close to the future real case. The case of a $50 \mathrm{MW}$ offshore wind farm in China, established in 2016, was studied with a genetic algorithm (GA) to identify the viability of the proposed formulation. 


\section{Time-cost-quality (TCQ) in offshore wind farm construction management}

\subsection{Importance}

Large scale offshore wind farms consist of offshore foundations, platforms and turbine towers which must tackle numerous obstacles during the construction period and thus higher cost and longer time are required to achieve the desired quality (Thomsen, 2014). Due to the unique attributes of such projects, no reversals or amendments are allowed. Even if the quality of a completed project is up to standard, on-going maintenance must be extremely comprehensive. In addition, substandard construction work may also lead to negative impacts on the marine life and the ecosystem. Additionally, regardless of the difficulties and high standards for wind farm projects, the costs are highly focused. Profitability is always the greatest concern for both the investors and contractors. Good budgeting allows the best use of labour, materials and equipment and is cost effective. In addition, due to the unique climate in the offshore area, the construction period of a wind farm is strictly limited as construction is forbidden during flood or typhoon period. An effective control mechanism is necessary to uphold the project time, cost and quality during the planning phase and to ensure the progress of the project is smooth while meeting the multiple objectives, such as quicker progress, lower cost and higher quality.

\subsection{The problem}

The main objective of a construction project, so as the offshore wind farm project, is to make construction to meet the requirements of the contract with the lowest cost, higher quality and shorter time, in parallel with other targets comprehensively. Those multi-objectives are interrelated, interacted and inter-conflicted. As a result, the multiobjective trade-off problem among various components comes to be condition precedent at the project planning stage (Mohammadipour and Sadjadi, 2016). Beginning with a determination of construction activities, the planning phase attempts to put in place a reasonable and applicable construction plan and duration for the respective tasks. Then, a reasonable budget can be set to match the quality objectives. Conflicts among time, cost and quality of a project are, however, extensive and inevitable (Hajiagha et al., 2015). Higher quality requires a high level of support, such as excellent construction techniques, skilled labour and innovative materials, and this requires more effort and resources during construction to check and monitor the progress continuously, which may lead to a higher cost. In addition, the more time spent on the construction project, the more money required for daily expenses. Establishing a balance between project timing, cost and quality is thus a critical issue (Babu and Suresh, 1996).

\subsection{Previous solutions}

As a common project management issue, this multiobjective problem involving the project TCQ trade-off has been addressed by project management experts while many efficient and effective models to simulate this kind of problem have been proposed (Zhang et al., 2014). The problem continues to be examined by scholars in various fields. However, none has provided solid evidence for offshore wind farm projects.

To solve the general construction problems in a residential setting, Hajiagha et al. (2015) developed a series of mathematical models for a continuous TCQ tradeoff which estimated the parameters of the project activity uncertainty with grey numbers. However, the precision of the simulation mostly relies on the correction of these parameters while the definition of the grey numbers is highly dependent on the experiences of the project managers involved over long terms, and it is too specialised to be understood by non-experts. Thus, Vahid Faghihi et al. (2014) presented a hands-on model of time-cost-quality trade-off problem (TCQTP) which aimed at generating more effective project networks and schedules without the need for on-site experience, planning ability and oversight. They considered the project scheduling to be the core of TCQTP and integrated it with virtual three-dimension geometric modelling. This type of transformation involves geometric elements in a 3D model, with activities to be scheduled and presented as nodes, and the dependencies between each activity as columns and beams. This method reduces the effect of experience but introduces more difficulties in the transformation of the model into threedimensional geometry. In this study, the valid cost and time which differ from grey numbers and are easier to acquire and collect, are put into analysis. This method depends less on the experience of individuals and has less bias from personnel influence. It also produces a more objective and reliable result for decision-making.

Despite traditional objectives which stuck on time, cost and quality, several other objectives are proposed to achieve a more efficient monitoring of the construction process. Afruzi et al. (2014) proposed a trade-off model in terms of two kinds of activity performance modes: normal and crush. Specifically, the crush mode appears to lead to shorter time requirement but has a higher direct cost with more resources required and poorer quality than those of the normal mode. Three objective functions were developed based on these two performance modes. However, the cost objective function is simply choosing the direct cost rather than the total cost of the activity and the project time is treated as the sum of all activities. The activities of a project are treated as a sequence, ignoring the interactions with preceding activities. In contrast, Narayanan and Suribabu (2014) established a cost model by dividing the total project cost into direct and indirect costs; and the quality model considered the influence of processor activity by 
quality indicators. However, the models are proposed for the management of on-going projects and serve for main contractors only. Mohammadipour and Sadjadi (2016) provided a trade-off model among higher cost, higher risk and poorer quality when facing the client's requirement on crushing construction schedule. The multi-objectives are isolated to three single objective functions, which indicate the floating value of risk, quality and cost. By applying the enhancement of the risk, deduction of quality and increase of the cost, it could be easier to quantify the change value of the risk and quality. However, similarly, quantifying method applied constraint to the model's applicability and flexibility into the in-progress projects only.

In addition, researchers proposed dozens of methods to simulate the interaction between the time, cost and quality. Zhang et al. (2014) used the critical path method (CPM) to monitor the schedule of the project, and the quality performance index (Q) is proposed to quantify the individual quality of each activity in a project. $\mathrm{Hu}$ and He (2014) proposed a trade-off model based on a work breakdown structure. They divided a construction project into a series of activities with respect to the construction labour, materials, equipment and administration. The breakdown based models provide a brief analysis of each activity in a project and make it possible for managers to modify the design in detail. However, it is impractical for a project manager to deal with all activities at such a detailed level. Despite the simultaneous activities in the resource allocation, the total duration of the project is overestimated, and the effects of the preceding activities, in terms of total quality, are not monitored. Recently, Lim et al. (2015) set up a time-cost-quality-trade-off (TCQT) model according to the satisfaction of the parties involved in the construction project. Evidential reasoning was first used in the multi-criterion decision-making (MCDM) approach of Monghasemi et al. (2015), who proposed a comprehensive framework to integrate MCDM methods with optimisation techniques. They combined a multiobjective genetic algorithm (MOGA) and non-dominated sorting genetic algorithm-II (NSGA-II) to solve a discrete TCQTP. Tran et al. (2015) applied a minimum ratio method to combine parameters for time, cost and quality into one objective function, and by solving this objective function, the trade-off problem could be consequently solved. Zhong et al. (2017) applied the on-site monitoring system to obtain the real-time quality of the on-going structure, in order to analyse the dynamic motion of the construction quality. However, almost all cases studied in these researches are applied on small or medium scaled residential projects, which are relatively standard and related to smaller scales of variation.

Instead, Zhong et al. (2017) applied the trade-off model established to the rock fill dam project during its construction process with dynamic in-situ monitoring system. Aminbakhsh and Sonmez (2016) applied the proposed discrete time-cost trade-off model on all small, medium and large-scale projects by tuning the diameter settled during the running and processing of the particle swarm optimisation method. However, none of these methods have been applied to offshore wind farm projects and the applicability and effectiveness for this kind of project has never been verified.

A literature review has identified multiple methods that were developed to solve the proposed model, and based on these endeavours, this study aims to establish a more systematic and applicable set of models to simulate the interaction among TCQ, with the purpose of simplifying the pre-construction planning for offshore wind farm projects. Models are selected and proposed with their merits to simulate the interaction among time, cost and quality. With easier control for project executors and managers, less dependence is required on experts' experiences and a result closer to the final project construction. CPM is applied to sum up the total time for project construction (Fu and Zhang, 2014); the work breakdown theory (WBT) divides the total cost into individual activities, each with their own direct, indirect and tardiness costs ( $\mathrm{Hu}$ and $\mathrm{He}, 2014)$; and the indicator, the $\mathrm{Q}$ is proposed to ensure the project quality (Zhang et al., 2014). To achieve the optimisation of these three elements, the minimum ratio method (MRM) is used to combine them into one objective function. Only when the minimum value of this function is found, the corresponding value of the time is marked as the optimal solution, in addition to the indicated cost and quality.

All of the parameters required for establishing the model are relatively objective and there is no need for longterm construction experience. All of the models used to solve the trade-off problem are familiar and convincing after long-term development, to be understandable for executors and managers. The computational intelligence method of the GA is proposed for the optimisation of the proposed objective function.

\section{Mathematical modelling of the multi-objective TCQT}

To solve the multi-objective optimisation problem, this study separates the objectives and first finds the interrelationship functions for each objective, then to build an objective function to integrate them into one function. With the optimisation of the objective function, the optimal solution can be derived. This study begins by qualitatively analysing the relationship and form models between timecost and time-quality in a construction project, and uses the MRM to promote an objective function $f$, combining and fully considering all the linkages.

\subsection{Introduction}

Multi-objective problems are common in almost all of the decisions made in a project process. Therefore, finding a solution is critical for all managers in each hierarchy. 
The basic multi-objective problem model can be described below, where $x$ is the $\mathrm{N}$-dimension decision vector, $y$ is the objective vector, $f(x)$ are the individual minimum objective functions, $g(x)$ is the $j_{t h}$ constraint function and $S$ is the feasible solution region for the decision vector (Ulungu and Teghem, 1994).

$$
\begin{aligned}
& x=\left[x_{1}, x_{2}, \ldots, x_{n}\right] \\
& \min y=f(x)=\left\{f_{1}(x), f_{2}(x), \ldots, f_{n}(x)\right\} \\
& x \in S=\left\{x / g_{j}(x) \leq 0, j=1,2, \ldots, p\right\} .
\end{aligned}
$$

\subsection{Time modelling}

This study chooses the duration of each activity as the basic element for calculation, together with a Gantt chart, to determine the overall duration of the project.

\subsection{Time-cost modelling}

Since the development of the scheduling network, many practical mathematical models have been proposed based on an analysis of the link between the duration of the activities and the total cost for the accomplished activities, such as the linear time-cost relationship model (Perera, 1980) in which the linear programming is easy to display but cannot accurately simulate a real case and the discrete time-cost trade-off model which is closer to real cases but harder to calculate. Thus, a power function with several indicators is applied in this study to simulate real cases more accurately than the linear model, but is easier to calculate than the discrete model.

The proposed relationship between time and cost is presented in Figure 1, where $b_{i j}$ is the lowest cost of the activity, $T_{S}$ is the shortest duration of the activity, $T_{N}$ is the normal duration of the activity, and $T_{L}$ is the longest duration of the activity (Zhang et al., 2014). The total cost of construction is usually broken down into the direct cost, the indirect cost and the tardiness cost, which can be added together for each individual activity.

\subsubsection{Direct cost}

The direct cost includes the direct labour, material and equipment, together with bills to subcontractors. In this study, the increasing marginal direct cost coefficient $\gamma_{i}$ is used to consider the construction environment and level. If the construction materials are easy to obtain, the construction level will increase with the rising level of labour and $\gamma_{i}$ will be small; if the construction materials are not easy to obtain, $\gamma_{i}$ will be relatively large. The duration of the activity is inversely proportional to $\gamma_{i}$. The formula of can be presented as:

$$
D C=\sum_{i=0}^{\mathrm{n}}\left[b_{i}+\gamma_{i}\left(T_{i}^{R}-T_{i}^{I}\right)^{2}\right]
$$

where $D C$ is the direct cost, $b_{i}$ is the direct cost of the activity $i$ in normal duration, $i=1,2, \ldots n$, for all the activities of a project, $\gamma_{i}$ is the increasing marginal direct cost coefficient, $T_{i}^{R}$ is the real duration of the activity $i, T_{i}^{R} \in\left[T_{i}^{S}, T_{i}^{L}\right] ; T_{i}^{S}$ is the shortest duration of the activity $i$, $T_{i}^{L}$ is the longest duration of the activity $i$, and $T_{i}^{I}$ is the initial duration of the activity $i$ (proposed in the contract). This formulation shows that the cost of the project will increase in its power function with fluctuations in the project duration.

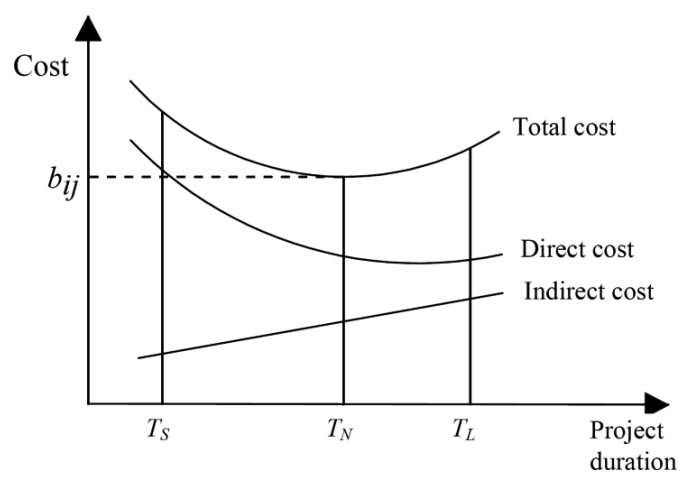

Figure 1. Relationship between the project duration and cost.

\subsubsection{Indirect cost}

The main element of the indirect cost is the manufacturing costs, which contain the indirect labour cost and depreciations, and is often calculated by multiplying the rate of $I C$ with $D C$. This rate is proposed in the contract according to the project evaluation. In China, taxes are an important element and are proportional to the construction profit and the indirect cost.

$$
\begin{aligned}
& I C=T \frac{I C_{0}}{T_{0}}, \\
& I C_{0}=D C_{0} \times r_{I C},
\end{aligned}
$$

where $I C$ is the indirect cost, $T$ is the real duration of the project, $I C_{0}$ is the indirect cost at the planned initial duration, $T_{0}$ is the initial duration of the project, $D C_{0}$ is the direct cost at the planned initial duration, and $r_{I C}$ is the rate of indirect cost.

\subsubsection{Tardiness cost}

The tardiness cost is a condition listed in the contract and is the reward or punishment for being ahead of or delaying the proposed schedule. In this study, the formula proposed by Deckro et al. (1995) was used, where $c_{1}$ is the coefficient of reward for early completion (\$ per day).

$$
T C= \begin{cases}c_{1}\left(T_{0}-T\right), & \& T \leq T_{0} \\ \left(e^{\frac{T-T_{0}}{T_{0}}}-1\right)(D C+I C), & \& T>T_{0}\end{cases}
$$


To conclude, the target minimum total cost of a project can be shown as follows:

$$
\operatorname{Min} C=D C+I C+T C \text {. }
$$

With reference to the concept of marginal utility in economics theory, these time-cost models are presented in the power function together with a new indicator. Real cases are in fact much more complex than the formula presented in Equation (3), because the tardiness rules are always factored into the cost when completing a project. Equation (6), which combines the direct and indirect costs with the tardiness cost, is thus much closer to the realistic conditions of construction projects and therefore it is treated as the basis formula for the time-cost model.

\subsection{Time-quality modelling}

In terms of the breakdown activities, researchers traditionally found quality to be proportional to the schedule of an activity for calculation convenience (Pollack-Johnson and Liberatore, 2006). They assumed that the activity quality is $100 \%$ when the activity is completed in its normal time frame. However, this study uses a more practical method for simulating the mathematical link between the quality and the duration of a single activity, as it is suggested that the quality will in fact reduce with the extension of the activity time. It is assumed that the relationship should be treated as a quadratic one, which is more in line with real cases, and $Q$ is used to quantify the quality of the project (Zhang et al., 2014). The $Q$ of each single activity can then be defined as follows:

$$
Q_{i}=a_{i} t_{i}^{1}+b_{i} t_{i}+c_{i}
$$

where $t_{i}$ is the duration of the activity $i ; a_{i}, b_{i}, c_{i}$ are the coefficients of the quadratic equation, which can be derived from known information as in Figure 2; $T_{S}$ is the shortest duration of the activity; $T_{B}$ is the best duration of the activity, and can be derived by:

$$
T_{B}=T_{S}+0.618\left(T_{L}-T_{S}\right),
$$

where $T_{L}$ is the longest duration of the activity.

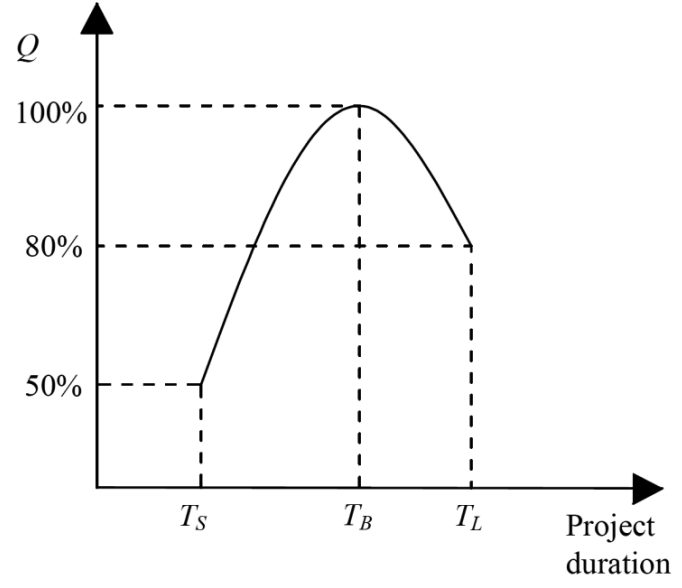

Figure 2. Relationship between the project schedule and quality.

In addition, the total quality of a project is determined by the quality of each individual activity within a designated schedule. Interactions among individual activities will have strong effects on the total quality. Researchers suggested evaluating the total quality by combining the quality of the individual activities with a quality importance weighting. However, this method requires more on-site working experience and a large amount of work. Thus, a new method was proposed by Zhang et al. (2014) that involves applying a project network to monitor the interaction between sequential activities. Since the whole project quality is closely connected to each single activity, and each following activity $i$ is affected by its predecessor $j$, this study updated the processing of the quality output of each activity as:

$$
Q^{\text {out }}=\left[1-\prod_{j=1}^{n}\left(1-Q_{j}^{\text {in }}\right)\right] \times Q_{i},
$$

where $Q_{i}$ is the quality of the predecessor activity $j(j=1,2, \ldots, m, m$ is the number of predecessors of activity $i$ ). The final output is the $Q^{\text {out }}$ of the whole project.

Compared to the traditional importance weight method, the new $Q$ method uses the quadratic function that better fits the actual circumstances of a construction project. This method can then derive the whole project quality from the individual quality of the activities, rather than confirming the importance weights of every activity, which is more natural and accurate if manual experience is limited.

\subsection{Multi-objective mathematical function}

In addressing multi-objective problems, a mathematical formulation is typically developed to quantify problems and solve them by mathematical optimisation. The idea is to generate an objective function and find the optimal solution of this function. Many methods can be used to generate the objective function, such as the linear 
weight sum method and the weighted averages method. This study uses the MRM to formulate the objective function $f(x)$, and by finding the minimum value of $f(x)$, the measurement indicator $T$ can be obtained and the best trade-off between our models can be found:

$$
\begin{aligned}
& f(C, T, Q)=\left(\omega_{c} \frac{C}{C_{0}}+\omega_{T} \frac{T}{T_{0}}+\omega_{Q} \frac{Q_{0}}{Q}\right) /\left(\omega_{c}+\omega_{T}+\omega_{Q}\right), \\
& C=C(T), \\
& Q=Q(T),
\end{aligned}
$$

where $\omega_{c}, \omega_{T}, \omega_{Q}$ are the relative importance indices of cost, time and quality of the project, respectively.

\section{Case study}

In this study, an actual offshore wind farm engineering project is used to verify the proposed models and the computational intelligence of the GA is used to assist in the solving process. The case is a $50 \mathrm{MW}$ offshore wind farm with ten $5 \mathrm{MW}$ turbines located in Putian, Pinhai Harbour, Fujian Province, China, which is approximately $12 \mathrm{~km}$ from Pinghai. Basic information about the Putian offshore wind farm project is presented in Table 1, where $b_{i}$ is the direct cost of the activity $i$ in normal duration; $i=1,2, \ldots n$, for all the activities of a project; $\gamma_{i}$ is the increasing marginal direct cost coefficient; $T_{i}^{S}$ is the shortest duration of the activity $i$; $T_{i}^{L}$ is the longest duration of the activity $i$; and $T_{i}^{I}$ is the initial duration of the activity $i$ (proposed within the contract). $T_{i}^{S}, T_{i}^{I}$ and $b_{i}$ are given in tenders from the contractor, and $\gamma_{i}$ is also derived from the tender. The initial schedule of this project can be derived as

\begin{tabular}{|c|c|c|c|c|c|c|c|}
\hline ID & Activities & Predecessors & $\begin{array}{c}\text { Initial cost } \\
\left(b_{i}\right)(¥)\end{array}$ & $\begin{array}{c}\text { Increasing direct } \\
\text { cost margin } \\
\text { parameter }\left(\gamma_{i}\right)\end{array}$ & $\begin{array}{c}\text { Shortest } \\
\text { duration } \\
\left(T_{i}^{S}\right) \text { (days) }\end{array}$ & $\begin{array}{l}\text { Initial duration } \\
\left(T_{i}^{I}\right) \text { (days) }\end{array}$ & $\begin{array}{c}\text { Longest } \\
\text { duration } \\
\left(T_{i}^{L}\right) \text { (days) }\end{array}$ \\
\hline 1 & Preparation & & $25,527,460.00$ & 0.7000 & 10 & 15 & 20 \\
\hline 2 & Temp. work establishment & 1 & $10,273,470.00$ & 0.8000 & 15 & 18 & 30 \\
\hline 3 & Foundation construction & 2 & $23,971,430.00$ & 0.9000 & 90 & 93 & 135 \\
\hline 4 & Piling works & 1 & $60,441,283.00$ & 1.000 & 90 & 119 & 160 \\
\hline 4.1 & $\begin{array}{l}\text { Manufacture of } \\
\text { dia. } 1.9 \mathrm{~m} \text { steel piles }\end{array}$ & 1 & $41,224,943.00$ & 2.9818 & 40 & 50 & 68 \\
\hline 4.2 & $\begin{array}{l}\text { Installation of dia. } 1.9 \mathrm{~m} \\
\text { steel piles }\end{array}$ & 2 & $12,286,130.00$ & 2.2143 & 20 & 29 & 40 \\
\hline 4.3 & Concreting & 4.2 & $6,930,210.00$ & 1.1237 & 40 & 56 & 70 \\
\hline 5 & Construction of piling platform & 2 & $45,883,324.00$ & 0.6160 & 90 & 113 & 210 \\
\hline 5.1 & Fabrication of steel casing & 2 & $3,993,520.00$ & 1.9461 & 30 & 36 & 50 \\
\hline 5.2 & Line 1 construction & 2 & $11,975,442.00$ & 3.4234 & 100 & 110 & 188 \\
\hline 5.2 .1 & \#9 Station & 2 & $3,005,982.00$ & 1.4056 & 70 & 110 & 130 \\
\hline 5.2 .2 & \#10 Station & 4.2 & $4,484,730.00$ & 3.6604 & 35 & 38 & 65 \\
\hline 5.2 .3 & \#8 Station & 4.2 & $4,484,730.00$ & 1.0190 & 35 & 43 & 65 \\
\hline 5.3 & Line 2 construction & 2 & $7,490,712.00$ & 3.9839 & 43 & 104 & 160 \\
\hline 5.3 .1 & \#4 Station & 2 & $3,005,982.00$ & 0.2315 & 35 & 104 & 120 \\
\hline 5.3 .2 & \#2 Station & 2 & $4,484,730.00$ & 1.1316 & 35 & 38 & 50 \\
\hline 5.4 & Line 3 construction & 2 & $22,423,650.00$ & 1.4480 & 82 & 82 & 120 \\
\hline 5.4 .1 & \#6 Station & 2 & $4,484,730.00$ & 2.4919 & 35 & 47 & 65 \\
\hline 5.4 .2 & \#1 Station & 2 & $4,484,730.00$ & 0.2449 & 35 & 60 & 65 \\
\hline 5.4 .3 & \#3 Station & 2 & $4,484,730.00$ & 0.4316 & 35 & 39 & 65 \\
\hline 5.4 .4 & \#7 Station & 2 & $4,484,730.00$ & 2.8659 & 35 & 60 & 65 \\
\hline 5.4 .5 & \#5 Station & 2 & $4,484,730.00$ & 1.2766 & 35 & 39 & 65 \\
\hline 6 & $\begin{array}{l}\text { Bearing steel, steel ladder, } \\
\text { handrail construction platform }\end{array}$ & 5 & $2,610,750.00$ & 0.6500 & 45 & 61 & 85 \\
\hline 7 & $\begin{array}{l}\text { Cap rubber fender system } \\
\text { installation construction platform }\end{array}$ & $\mathrm{m}$ & $4,500,000.00$ & 1.5385 & 45 & 46 & 65 \\
\hline 8 & Submarine cable laying & 5 & $14,567,960.00$ & 0.6500 & 59 & 70 & 168 \\
\hline 8.1 & Underwater rock excavation & 5 & $165,322.00$ & 9.4873 & 35 & 44 & 45 \\
\hline 8.2 & Submarine cable installation & 8.1 & $10,813,654.00$ & 4.1507 & 35 & 43 & 45 \\
\hline 8.3 & $\begin{array}{l}\text { Cement sandbags scour } \\
\text { protection layer }\end{array}$ & 8.2 & $3,588,984.00$ & 1.9511 & 20 & 43 & 45 \\
\hline 9 & Wind fan installation & $5,6,7,8$ & $50,716,870.00$ & 108.0683 & 122 & 140 & 250 \\
\hline 9.1 & $\# 1$ and \#2 fans installation & $5.3,5.4$ & $10,143,374.00$ & 0.0149 & 20 & 30 & 75 \\
\hline 9.2 & $\# 3,4,5,6$ fans installation & $5.3,5.4$ & $20,286,748.00$ & 3.0130 & 20 & 59 & 85 \\
\hline 9.3 & $\# 7,8,9,10$ fans installation & $5.2,5.4$ & $20,286,748.00$ & 1.7133 & 20 & 37 & 85 \\
\hline 10 & $\begin{array}{l}\text { Fans commissioning and } \\
\text { operation }\end{array}$ & 9 & $4,200,000.00$ & 10.2641 & 100 & 119 & 200 \\
\hline 11 & Project checking and accepting & 10 & $60,000.00$ & 7.0588 & 10 & 12 & 20 \\
\hline & Sum & & $242,752,547.00$ & - & - & - & - \\
\hline
\end{tabular}
shown in Figure 3.

Table 1. Initial information about the wind farm project. 


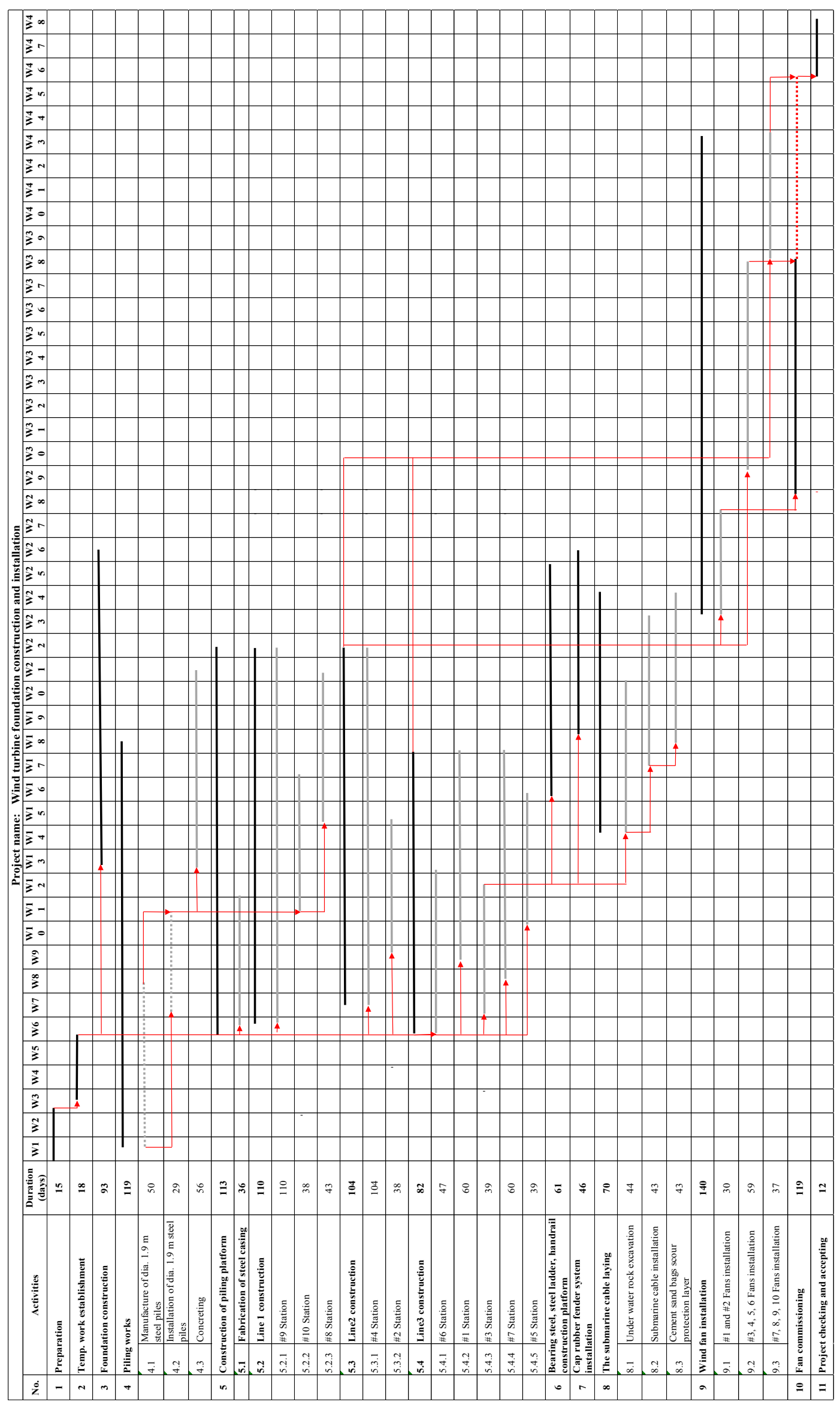




\subsection{Case background}

Initial cost: According to the formula provided and the tax payment criteria in China, the rate of the indirect cost is assumed to be $20 \%$, the firm profit tax rate is $8 \%$ and the comprehensive tax rate is $3.41 \%$. The initial cost can then be calculated as follows:

$$
\begin{aligned}
& D C_{0}=¥ 242,752,547, \\
& I C_{0}=¥ 242,752,547 \times 20 \%=¥ 48,550,509.40, \\
& R_{0}=\left(D C_{0}+I C_{0}\right) \times 8 \%=¥ 23,304,244.51, \\
& C_{0}=\left(D C_{0}+I C_{0}+R_{0}\right) \times(1+3.14 \%)=¥ 325,335,409.87 .
\end{aligned}
$$

Initial time: According to the Gantt chart in Figure 4 , the initial schedule for this project is 334 days, so $T_{0}=334(d)$.

Initial quality of project: Theoretically, the initial $Q$ should be $100 \%$, but after the calculation with the proposed models, the $Q$ of the project is $77.32 \%$.

Importance index: In this study, a questionnaire was prepared with questions to find the relative importance level of time, cost and quality for a large-scale construction project in different phases of planning and constructing from the construction management. After collecting 211 questionnaires, with a $50 \%$ feedback rate from those involved in the construction industry all around China, the importance index of cost, time and quality is determined to be 1.2336, 1 and 1.0456, respectively, which means that most of the participants believe that the cost is of the highest importance to a project, followed by quality, then time. Therefore, $\omega_{C}=1.2336, \omega_{T}=1$, and $\omega_{Q}=1.0456$.

\subsection{Model establishment}

This case is a multi-objective problem, which attempts to optimise the project duration in parallel with cost reduction and quality improvement, under the criteria defined in the contract. The multi-objective optimisation models in this case can be presented as follows, when accounting for the initial conditions:

1. Cost optimisation model. The models could be referred in Equations (2) - (6).

2. Quality optimisation model.

$$
Q=G_{i=0}^{n} Q_{i}=Q_{n}^{\text {out }}
$$

G - Gathering of all the activities of the project.

3. Time optimisation model.

$$
\operatorname{MinT}=\sum_{(i, j) \in G} t_{i j}
$$

where $t_{i j}$ is the time duration of the activities in the critical path of the project (El-Rayes and Kandil, 2005).

4. Multi-objective fitness function. The function could be referred in Equation (10).

\subsection{Solution with $G A$}

In this study, a mature computational intelligence algorithm known as the GA is applied to confirm the solution found by the proposed model (Gen et al., 2008). The duration of each activity in the project is set as the variable vector, and there are 34 variables. The initial population size was set at 100 and generated randomly, and the termination rule was set at 5,000 as the maximum generation and the real number encoding method was applied in MATLAB. The progress of the solution and the main programme code can be described as follows:

Step 1. Recognition of the optimisation problem, giving a definition of the fitness function as shown in Equation (10) and setting the running parameters as follows: the dimension of the feasible solution is $D=34$; the feasible solution domain is given as $\left[x_{\min }, x_{\max }\right]^{D}$, where $x_{\min }$ is the allowable shortest duration and lower bound of this project; and $x_{\max }$ is the allowable longest duration and upper bound of the project; population size $N=100$; the generation $g_{\max }=10,000$; the stall generation limit is given as 50; the constraint tolerance and function tolerance are both set as $(1 e-20)$; and the initial penalty and penalty factor are 1.

Step 2. Initialisation. This phase has two parts. First, the initial information $T_{0}, C_{0}, Q_{0}$ of the case problem is produced, according to the initial accomplishment duration of the project and the fitness function $f$. Second, the initial population $P_{1}$ is randomly generated $(g=1)$. As presented in Step $1, P_{1}$ contains 100 solutions with 104 genes and represents the feasible duration of all the activities in the project.

Step 3. Evaluation. The fitness scores of the initial generation are computed by monitoring the fitness function presented in Equation (10) and assessments are made of the survival possibility of these feasible solutions. After evaluating the fitness score of the feasible solutions, the parts of the solutions with good performance and relatively high fitness scores are chosen to be a parent chromosome for the regeneration of new generations and to update the population. Chromosomes with bad performance are simultaneously eliminated.

Step 4. Reproduction. The parent population is the operator for crossover and mutation to re-arrange the genes of the solutions, thus producing offspring solutions.

Step 5. After evaluation based on the fitness function, a selection operation is conducted to select the high fitness solutions from both the parent and child populations and then the selected solutions are reunited as a new combined population, accomplishing an update of the population. Steps 3 - 5 are repeated until the termination criteria of 10,000 times that the generation number is met.

Step 6. After 10,000 generations, the best fitness solution is obtained, which is the so-called optimal solution.

The evolution process is shown in Figure 4. 


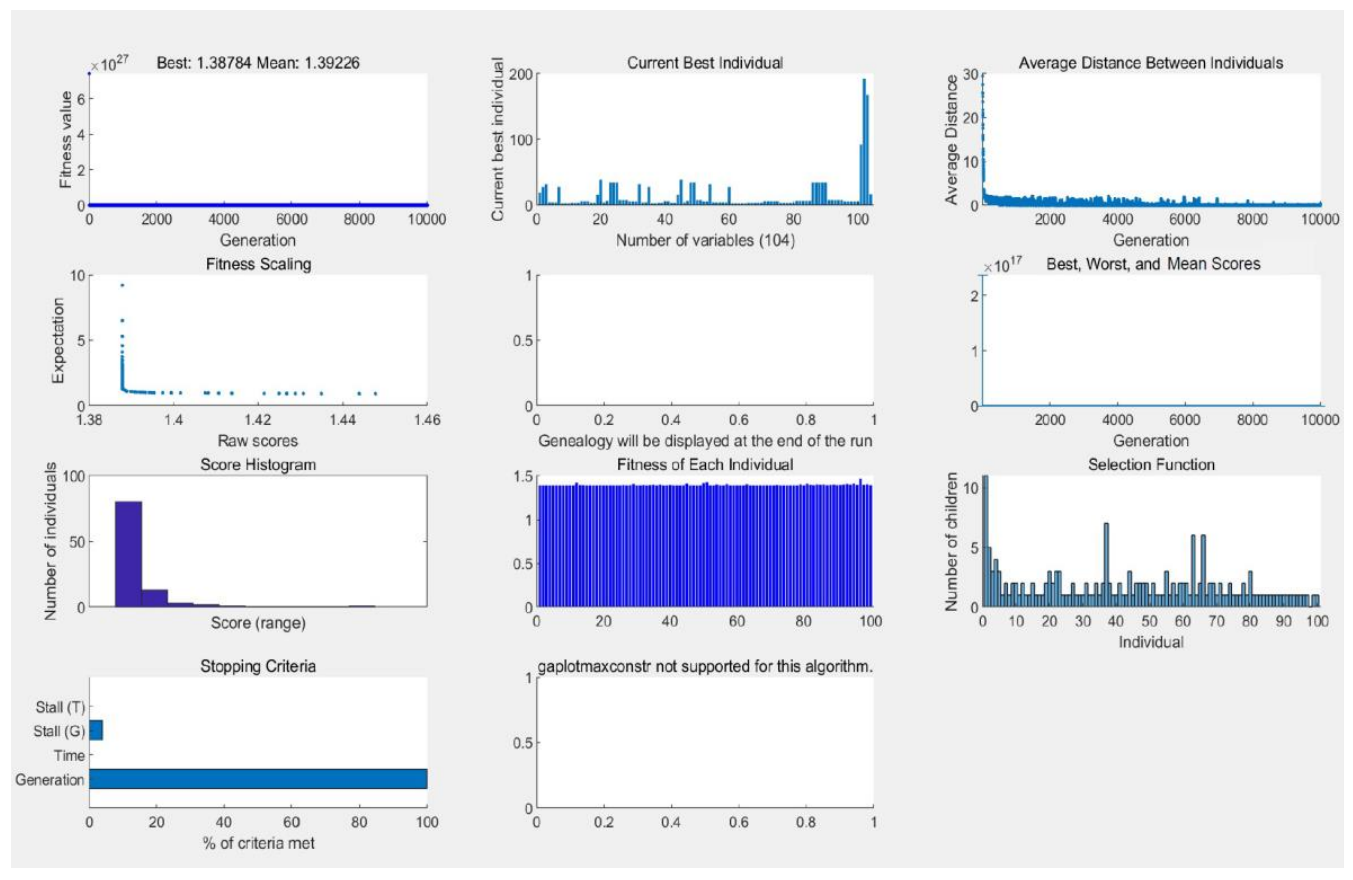

Figure 4. Evolution process by a GA for the wind farm project.

\subsection{Results analysis}

It is a practical method to evaluate the practicability and reliability of the optimisation process by directly comparing the actual data and the optimal output. The closer the distance between optimal output and the actual data is, the more useful the optimal process is. In this case, after running the GA, the final results are as follows:

As Figures 5 - 7 show, the quality of the project is found to be high and approaching the value 1 with a $0.01 \%$ increase in cost. The duration of the project is extended to improve the project quality but is still within the contractrequired period. Additionally, when compared to the actual completed project, the bias between the programme optimising and the real-case optimising by experts is reasonable, with the deviations in duration, cost and quality at $5.76 \%,-0.0040 \%$ and $0.02 \%$, respectively. These data mean that the time, cost and quality, as optimised by the programme, are closer to the real project, as constructed and monitored by experts, than to the original planning scheme. After applying the proposed mathematical models to an offshore wind farm project, it is apparent that the multi-objective optimisation is constructed reasonably well and is close to the real case results. It provides the possibility for the project manager to acquire a clearer and more accurate prediction of the future project in terms of project time, cost and quality at the pre-construction stage. It can be concluded that this type of optimisation for the TCQ of a project is practical and could be applied to assist in improving the planning and management of the wind farm project. In addition, with different importance priority parameter settings for the project time, cost and quality, this

mathematical model is sufficiently flexible for managers to achieve optimisation under different requirements.

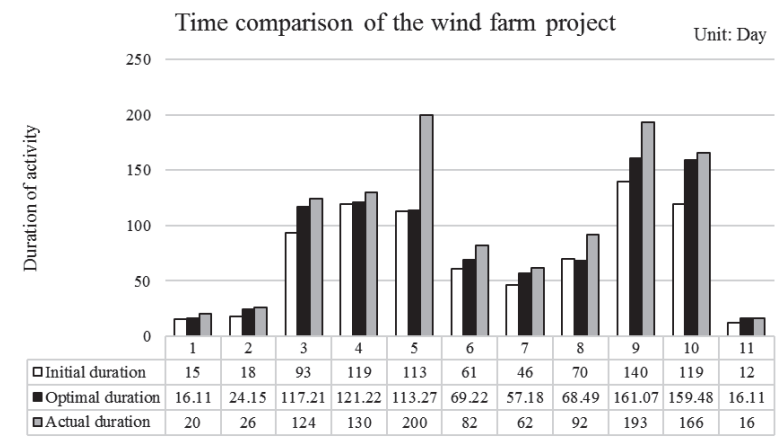

Figure 5. Time comparison of the wind farm project.

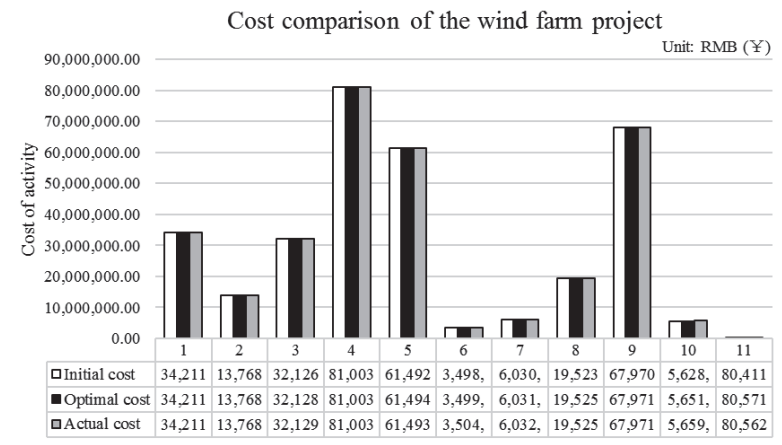

Figure 6. Cost comparison of the wind farm project. 


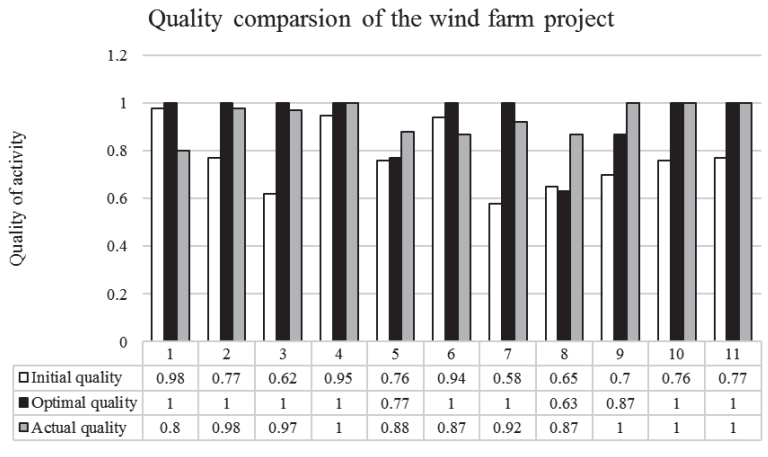

Figure 7. Quality comparison of the wind farm project.

\section{Conclusion}

The urgent requirement for renewable and sustainable green energy generation has led to the rapid development and application of wind power generation techniques world-wide. As additional wind farm projects are likely to be established, the planning of their construction must be defined and monitored to meet social requirements. In this study, a brief introduction to the background and significance of wind farm project construction planning and the multi-objective optimisation problem were provided. A summary and analysis of the research and achievements regarding the multi-objective problem are then presented, together with previous studies of the GA as a common problem solver. The relationships between project TCQ are then analysed. By comparing traditional models from other researchers and scholars, a series of general mathematical models for the three parameters was derived and a fitness function to transform the multi-objective problem into a single-objective problem was presented. Finally, in a case study, the proposed models are applied to an actual offshore wind farm project for verification. By running the real offshore wind farm project with the original project data in the proposed model and comparing the output with the actual values, it can be seen that the optimisation results are close to the actual case, and the proposed model is practical for improving the accuracy of the project condition prediction during the pre-construction stage in terms of time, cost and quality. Additionally, since the data and the applied parameters are objective and there is no need to perform much data analysis, flexibility exists for managers in execution and monitoring. It is a simple but efficient tool for managers to control. In addition, because of the flexibility and data isolation, this trade-off model should be applicable in many other types of construction projects. However, this study still has many limitations. First, the established mathematical models for the TCQ trade-off are not perfect, as they cannot take all aspects of real-life projects into consideration, such as the climate, environmental effects and interactions between preceding and following activities. The interactions between activities have been considered as yes or no issues, while in reality, many activities can be started when the former activities are only partially completed. To improve and enhance the models, recommendations that could be applied in future studies were provided. Additional actual project conditions should be taken into consideration, such as the impact of climate and possible effects on the environment. Specific client preferences in terms of time, cost and quality should also be identified. Then, the interactions between the sequential activities should be presented more specifically. To conclude, suggestions for improvement and in-depth study would be appreciated, in the form of valuable advice from experts, teachers and students in the field.

\section{Acknowledgements}

The work described in this paper was fully supported by a grant from the Research Grant Council of the HKSAR, People's Republic of China [Project No. CityU 11257816].

\section{Notes on contributors}

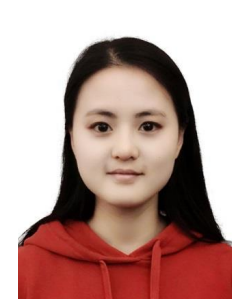

Miss Gloria Yushan Liu received her B.Eng. degree in Port, Waterway and Coastal Engineering and Dural Degree B.Mgt. in Accounting from Changsha University of Science and Technology, People's Republic of China and the M.Sc. degree with distinction in Civil and Architectural Engineering from the City University of Hong Kong (CityU). After graduation from CityU in 2015, she devoted herself into the construction industry as a Quantity Surveyor. During the four years of working in Hong Kong, she has been involved in many civil engineering projects, typically, including the 12106 New World Centre Remolding Project, MTRC Shatin to Central Link Project, the Airport Authority Hong Kong (AAHK) North Commercial District Enabling Works, Automated People Mover (APM) and Baggage Handling System (BHS) Tunnels on Existing Airport Island, and Airport Authority Midfield Aprons Development Work.

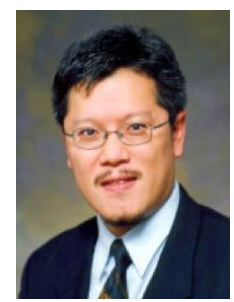

Ir Dr Eric Wai Ming Lee received his B.Eng. degree with first class honours in Building Services Engineering and the Ph.D. degree in Fire Engineering from the CityU. He is a Professional Engineer and currently the Panel Member of Fire Discipline of The Hong Kong Institution of Engineers (HKIE), the Assessor of Hong Kong Laboratory Accreditation Scheme of the HKSAR Government and also the member of various technical committees of the Buildings Department of the HKSAR Government. 


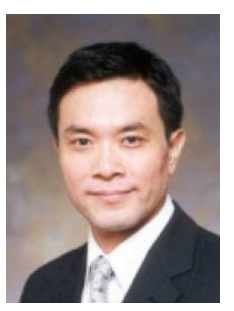

Ir Prof Richard Kwok Kit Yuen obtained his first degree in Mechanical Engineering at The University of Hong Kong and Ph.D. degree from the School of Mechanical and Manufacturing Engineering, University of New South Wales, Australia. He is currently the Professor and Head of the Department of Architecture and Civil Engineering, CityU. He is a Registered Professional Engineer and members of the Institution of Fire Engineers (IFE) (UK), the Society of Fire Protection Engineers (SFPE) (USA) and the HKIE. His research interests include fire safety and engineering, pyrolysis and combustion, applications of computational fluid dynamics (CFD), fire retardant materials, neural network modelling applications in fire engineering, Heating, ventilation, and air conditioning (HVAC) and control engineering. He is the Co-Principal Investigator (Co-PI) of the Theme-based Research Scheme project entitled "Safety, Reliability, and Disruption Management of High Speed Rail and Metro Systems" with over HKD 40 millions funded by the Research Grants Council of the HKSAR. He has published over 220 publications includ international journal articles and conference papers, and coauthored the book entitled: Computational Fluid Dynamics in Fire Engineering - Theory, Modeling \& Practice. Ir Prof Yuen had successfully supervised more than 20 Ph.D. graduate students and is supervising more than 10 $\mathrm{Ph}$.D. graduate students currently as the main supervisor. He has also been the examiner of many Ph.D. candidates both locally and internationally. He has been engaged in research consultancies and is also the Founding Member of the Disciplinary Advisory Panel of the Fire Discipline of the HKIE. He has served as members of Examination Board for Lift and Escalator Engineers of the Electrical and Mechanical Services Department and Inspector Registration Committee of the Buildings Department, the HKSAR Government, respectively. He is also currently the member of the Engineering Panel of the Research Grants Council of the HKSAR. He received The President's Award of CityU.

\section{References}

[1] Afruzi EN, Najafi AA, Roghanian E and Mazinani M (2014). A multi-objective imperialist competitive algorithm for solving discrete time, cost and quality trade-off problems with mode-identity and resourceconstrained situations. Computers \& Operations Research, 50, pp. 80-96.

[2] Aminbakhsh S and Sonmez R (2016). Discrete particle swarm optimization method for the largescale discrete time-cost trade-off problem. Expert Systems with Applications, 51, pp. 177-185.

[3] Babu AJG and Suresh N (1996). Project management with time, cost, and quality considerations. European Journal of Operational Research, 88(2), pp. 320-327.
[4] Carstensen J, Henriksen OD and Teilmann J (2006). Impacts of offshore wind farm construction on harbour porpoises: Acoustic monitoring of echolocation activity using porpoise detectors (T-PODs). Marine Ecology Progress Series, 321, pp. 295-308.

[5] Deckro RF, Hebert JE, Verdini WA, Grimsrud PH and Venkateshwar S (1995). Nonlinear time/cost tradeoff models in project management. Computers \& Industrial Engineering, 28(2), pp. 219-229.

[6] El-Rayes K and Kandil A (2005). Time-cost-quality trade-off analysis for highway construction. Journal of Construction Engineering and Management, 131(4), pp. 477-486.

[7] Faghihi V, Reinschmidt KF and Kang JH (2014). Construction scheduling using genetic algorithm based on building information model. Expert Systems with Applications, 41(16), pp. 7565-7578.

[8] Fu F and Zhang C (2014). A new approach to timecost-quality tradeoff problem for construction project. In: International Conference on Management Science \& Engineering 21th Annual Conference Proceedings, Helsinki: IEEE, pp. 1836-1842.

[9] Gen M, Cheng R and Lin L (2008). Network models and optimization: Multiobjective genetic algorithm approach. Germany: Springer Science \& Business Media. pp. 1-44.

[10] Hajiagha SHR, Akrami H, Hashemi SS and Mahdiraji HA (2015). An integer grey goal programming for project time, cost and quality trade-off. Inzinerine Ekonomika, 26(1), pp. 93-100.

[11] $\mathrm{Hu} \mathrm{W}$ and He X (2014). An innovative time-costquality tradeoff modeling of building construction project based on resource allocation. The Scientific World Journal, 2014, Article ID 673248.

[12] Klugmann-Radziemsk E (2014). Environmental impacts of renewable energy technologies. In: 2014 5th International Conference on Environmental Science and Technology. Singapore: IPCBEE, pp. 104-109.

[13] Lim TK, Jang WS, Choi JH and Lee DE (2015). Stochastic quality-cost optimization system hybridizing multi-objective genetic algorithm and quality function deployment. Journal of Civil Engineering and Management, 21(4), pp. 407-422.

[14] Mohammadipour F and Sadjadi SJ (2016). Project cost-quality-risk tradeoff analysis in a timeconstrained problem. Computers \& Industrial Engineering, 95, pp. 111-121.

[15] Monghasemi S, Nikoo MR, Fasaee MAK and Adamowski J (2015). A novel multi criteria decision making model for optimizing time-cost-quality tradeoff problems in construction projects. Expert Systems with Applications, 42(6), pp. 3089-3104.

[16] Narayanan AS and Suribabu CR (2014). Multiobjective optimization of construction project time-cost-quality trade-off using differential evolution algorithm. Jordan Journal of Civil Engineering, 159(3269), pp. 1-18. 
[17] Perera S (1980). Linear programming solution to network compression. Journal of the Construction Division, 106(3), pp. 315-326.

[18] Pollack-Johnson B and Liberatore MJ (2006). Incorporating quality considerations into project time/ cost tradeoff analysis and decision making. IEEE Transactions on Engineering Management, 53(4), pp. 534-542.

[19] Thomsen K (2014). Offshore wind: a comprehensive guide to successful offshore wind farm installation. Dutch: Academic Press. pp. 31-71.

[20] Tran DH, Cheng MY and Cao MT (2015). Hybrid multiple objective artificial bee colony with differential evolution for the time-cost-quality tradeoff problem. Knowledge-Based Systems, 74, pp. 176-186.

[21] Ulungu EL and Teghem J (1994). Multi-objective combinatorial optimization problems: A survey. Journal of Multi-Criteria Decision Analysis, 3(2), pp. 83-104.

[22] Zhang L, Du J and Zhang S (2014). Solution to the time-cost-quality trade-off problem in construction projects based on immune genetic particle swarm optimization. Journal of Management in Engineering, 30(2), pp. 163-172.

[23] Zhixin W, Chuanwen J, Qian A, and Chengmin W (2009). The key technology of offshore wind farm and its new development in China. Renewable and Sustainable Energy Reviews, 13(1), pp. 216-222.

[24] Zhong DH, Hu W, Wu BP, Li, Z and Zhang J (2017). Dynamic time-cost-quality tradeoff of rockfill dam construction based on real-time monitoring. Journal of Zhejiang University-SCIENCE A, 18(1), pp. 1-19. 